\title{
The Internet and its potential influence on suicide
}

\author{
Susan Thompson
}

The Internet is an uncensored interactive medium whose usage is growing exponentially - it is currently estimated that 171 million people from 135 countries use the Internet (Nua, 1999). This number doubles each year. creating a virtual community whose population will soon rival the size of any nation on earth. Internet access is highest among 18- to 24-yearolds. Of these, $37 \%$ are regular users, accessing the Internet at least once a week. As the medium has just been introduced into schools in the UK. this figure is likely to increase to close to $100 \%$.

The amount of information relating to suicide on the Internet is enormous and varied. A search using the keyword 'suicide' on any of the most popular search engines, typically yields 50 100000 entries. These range from web sites detailing particular individual and group suicides, for example, the suicides of Kurt Cobain. Michael Hutchence and the Heaven's Gate Cult, to sites relating research information on suicide, or advocating specific means of suicide. Entries relating to specific suicides are quite graphic, often containing copies of suicide notes, death certificates and colour photographs of the deceased. There are also electronic bulletin boards where suicide notes are posted, and access to chat rooms specifically devoted to the subject. Many of the relevant interactive web sites are 'pro-suicide', and forbid entries by those offering to dissuade users from committing suicide. Groups of 'suicide survivors' can also be accessed via the Net.

Presently, there is no opportunity on these sites to obtain access to mental health professionals or support groups who could respond directly with help to those contemplating suicide other than the Samaritans, who have entries and links to their main web site, (http://www.samaritans.org). In the first eight months of 1998, the Samaritans were contacted four million times, and 10000 of these contacts were by e-mail, a method favoured by young people, and one whose use has tripled in the last two years. Of those communicating by e-mail. $63 \%$ acknowledged suicidal feelings compared with $22 \%$ of those telephoning the Samaritans (Samaritans, 1998). Thus, the medium appears to have a specific ability to facilitate disclosure of suicidal ideas, particularly among young males under 35 , the group with the fastest rising suicide rate.

\section{Potential ways in which the Internet influences suicide}

The first case report detailing two suicide attempts in the UK prompted by information available on the Net has just been published (Haut \& Morrison, 1998). In this case, a female patient attempted to kdll herself by ingestion of wolfsbane (the most poisonous plant in the UK). and subsequently by injection of lighter fuel. necessitating surgical intervention. The authors were shocked at the number of web sites accurately detailing the fatal dose of commonly available substances, for instance paracetamol. which would cause death.

A further UK case report (Suresh \& Lynch, 1998), indicated how the Internet could be used to intervene in suicide attempts. In this instance, a woman who had taken a potentially lethal overdose and had posted a suicide note on the Internet was saved after a computer 'hacker' in the UK traced her whereabouts and alerted the local police, who broke into her apartment and took her to hospital. However, the ethical implications of these actions are obviously far reaching.

It is likely that the Internet has other effects on suicidal behaviour. Many studies (Berman, 1988), have reported a significant degree of clustering of suicides following media coverage of a suicidal event or personal contact with a suicide victim. Many of these suicides directly mimic the methods used by the initial individual who successfully completed suicide. These 'copycat suicides' are especially prevalent among the young. particularly if the initial victim was famous, or if the suicide provoked great interest. Thus, there is some evidence to suggest that if suicide is discussed publicly, some vulnerable individuals may consider it more readily.

There are certainly many news groups and bulletin boards on the Net which positively advocate suicide, and discourage individuals 
from seeking psychiatric or other help, dismissing it as worse than useless. For example, one newsgroup which calls itself The Church of Euthanasia', ( $w w w . e n v i r o w e b . o r g / c o e)$, suggests suicide as a positive act for all, and have announced their intention to set up a suicide assistance telephone hotline' to pursue this further. One of the most active newsgroups in this category is alt.suicide.holiday. This group was set up in the late 1980 s to discuss why suicides increase during holiday periods, but has evolved into a site for general discussion of suicide, with an alternative bulletin board for those contemplating committing suicide, or who wish to leave suicide notes. More than 900 messages are posted at this site every month, mostly from those considering suicide, or those who have attempted suicide in the past. The newsgroup specifies that messages of discouragement or religious disapproval will not be tolerated.

Baume et al (1997) followed three cases of individuals posting notes on this group's bulletin board over a period of three months in 1996. They found that the authors of these notices left a series of messages which were more like a presuicide diary than a traditional suicide note. Typically, a subject first stated his or her intent to commit suicide and asked other members of the group for information about the best method, and how to obtain any necessary items. In two out of the three cases studied, following the receipt of very specific relevant information (e.g. precisely how to aim a pistol in the mouth for maximum effect) along with messages of encouragement and support, the suicides were apparently successful via the use of firearms and selfpoisoning respectively. In the first case studied, discussion of the suicide continued thereafter on the bulletin board, with members in disagreement as to whether the encouragement he received from the group was actually appropriate. The third case studied did not succeed in his attempt to kill himself by carbon monoxide poisoning, and was subsequently admitted to a psychiatric hospital, having been found in a semi-conscious state. He discouraged others in the group from using this method, but did not comment on whether he found a hospital admission helpful.

Baume et al (1997) highlighted their concerns about the potential effects of such sites on the Internet. They noted the ambivalence of the notes posted by some subjects, and how their resolve strengthened following the encouragement of others, eventuating in successful suicides in some cases. They also felt there was evidence that vulnerable individuals were compelled so strongly by others that to back out or seek help would involve losing face.

These authors also felt there was evidence of a group death instinct at such sites. They cite research demonstrating the ability of street crowds to encourage suicidal individuals to jump off a building, and speculate about how much stronger such a group effect must be when all individuals involved are pro-suicide. Given the ambivalence which appears to exist in the cases they studied prior to suicide, the impact of such an effect is likely to be great. The phenomenon of cult or group suicides supports such a view.

Baume et al (1997) also note that young individuals are likely to be most vulnerable to these influences. Individuals who access the Net are qualitatively different to other individuals. Research already indicates they are psychologically more vulnerable, with higher risk taking behaviour, substance misuse and depression scores than control subjects (Martin, 1996). Most are also 18-24 year olds, a group with a high suicide rate and little peer support. A telephone survey of 1008 individuals over 16 years of age conducted by the Samaritans in 1996 revealed that young people did not understand depression and suicidal behaviour, and so were unsympathetic towards it (Samaritans, 1996). Although $26 \%$ of those interviewed knew someone who had committed suicide, over half the sample thought those who felt that people with depression "should pull themselves together". With these prevailing attitudes, it is unsurprising that young people turn to the Internet for support, with potentially disastrous effects.

Some authors advocate use of the Internet to gain support when feeling suicidal. For example, Miller \& Gergen (1998), who analysed all 232 entries on the AOL (America Online) suicide bulletin board for 11 months from November 1994 by type of communication. found the majority of entries were positive, inasmuch as they provided support, although there was little evidence that they changed the beliefs of those participating.

\section{Discussion}

The existing literature clearly indicates the complex effects of the Internet on suicidal behaviour. Young people appear particularly vulnerable in this respect, which raises ethical issues about whether this medium could or should be censored in some way - parents or schools might lock certain Internet sites if they were aware of the contents. The Internet could potentially be used to trace vulnerable individuals contemplating suicide, or having just attempted suicide. At present, this is unlikely to happen as suicide is considered a free choice in a liberal society. However, given the Suicide Act of 1951 prohibits others from encouraging suicide. there could be legal grounds for attempting to intervene at these sites. As reducing the suicide 
rate is one of the Health of The Nation key targets (Department of Health, 1992), it could be argued that interventions are justified for public health reasons.

The Internet also seems to facilitate a striking degree of self-disclosure which could be beneficial in identifying and communicating with those at risk of suicide. Given the exponential growth of this medium, it certainly seems that further research in this area is required to analyse the potential beneficial and adverse effects of this medium, and develop more effective therapeutic interventions.

\section{References}

Baume, P., Cantor, C. \& Rolfe, A. (1997) Cybersuicide: the role of interactive suicide notes on the internet. Crises, 18, 73-79.

BERMAN, A. (1988) Fictional depiction of suicide in television films and imitation effects. American Journal of Psychiatry, 146, 982-986.
DePaRTMENT OF HEALTH (1992) The Health of the Nation: A Strategy for Health in England. London: HMSO.

HAUT, F. \& MORRISON, A. (1998) The Internet and the future of psychiatry. Psychiatric Bulletin, 22, 641-642.

MARTIN. G. (1996) The influence of television suicide in a normal adolescent population. Archives of Suictde Research, 2, 103-117.

MiLLER, J. \& GeRgen, K. (1998) Lfe on the line: the therapeutic potentials of computer-mediated conversation. Journal of Marttal and Family Therapy. 2. 189-202.

NuA (1999) http://www.nua.ie/

SAMARTANS (1996) Challenging the Taboo. Newport, Isle of Wight: J. Arthur Dixon.

- (1998) 1998 Statistics. Newport, Isle of Wight: J. Arthur Dixon.

SuRESH, K. \& LYNCH, S. (1998) Psychiatry and the www: some implications. Psychiatric Bulletin, 22, 256-257.

Susan Thompson, Senior House Officer in Child Psychiatry, Ealing, Hammersmith \& Fulham Mental Health NHS Trust, Children and Family Consultation Service, Windmill Lodge, Uxbridge Road, Southall UB1 3EU

\section{Visit the Royal College of Psychiatrists web site at: http://www.rcpsych.ac.uk}

\section{Information on:}

- up-to-date information on College books, reports, and journals

- $\quad$ provides order forms and links to related sites

- $\quad$ press and public initiatives

- conferences

- library services

- examinations

- postgraduate education

- the college research unit

\section{And for members, information on:}

- divisions, faculties, sections, SIGS, committees and more

For further information please e-mail Lucy Alexander at: lalexander@rcpsych.ac.uk 